\title{
Sensor Noise in LISA Pathfinder: In-Flight Performance of the Optical Test Mass Readout
}

M. Armano, ${ }^{1}$ H. Audley, ${ }^{2}$ J. Baird, ${ }^{3}$ P. Binetruy, ${ }^{3, *}$ M. Born, ${ }^{2}$ D. Bortoluzzi, ${ }^{4}$ N. Brandt,${ }^{5}$ E. Castelli, ${ }^{6}$ A. Cavalleri, ${ }^{7}$ A. Cesarini, ${ }^{8}$ A. M. Cruise, ${ }^{9}$ K. Danzmann, ${ }^{2}$ M. de Deus Silva, ${ }^{10}$ I. Diepholz, ${ }^{2}$ G. Dixon, ${ }^{9}$ R. Dolesi, ${ }^{6}$ L. Ferraioli, ${ }^{11}$ V. Ferroni, ${ }^{6}$ E. D. Fitzsimons, ${ }^{12}$ R. Flatscher, ${ }^{5}$ M. Freschi, ${ }^{10}$ A. García,${ }^{13}$ R. Gerndt, ${ }^{5}$ L. Gesa,${ }^{14,15,{ }^{*}}$ D. Giardini, ${ }^{11}$ F. Gibert, ${ }^{6,16}$ R. Giusteri, ${ }^{2}$ C. Grimani,${ }^{8}$ J. Grzymisch, ${ }^{1}$ F. Guzman, ${ }^{17}$ I. Harrison, ${ }^{18}$ M.-S. Hartig, ${ }^{2}$ G. Heinzel, ${ }^{2}$ M. Hewitson, ${ }^{2}$ D. Hollington, ${ }^{19}$ D. Hoyland, ${ }^{9}$ M. Hueller, ${ }^{6}$ H. Inchauspé, ${ }^{3,20}$ O. Jennrich, ${ }^{1}$ P. Jetzer, ${ }^{21}$ U. Johann, ${ }^{5}$ B. Johlander, ${ }^{1}$ N. Karnesis, ${ }^{3}$ B. Kaune, ${ }^{2}$ C. J. Killow, ${ }^{22}$ N. Korsakova, ${ }^{23}$ J. A. Lobo, ${ }^{14,15, *}$ L. Liu, ${ }^{6}$ J. P. López-Zaragoza, ${ }^{14}$ R. Maarschalkerweerd, ${ }^{18}$ D. Mance,${ }^{11}$ V. Martín, ${ }^{14,15}$ L. Martin-Polo, ${ }^{10}$ F. Martin-Porqueras, ${ }^{10}$ J. Martino,${ }^{3}$

P. W. McNamara, ${ }^{1}$ J. Mendes,${ }^{18}$ L. Mendes, ${ }^{10}$ N. Meshksar, ${ }^{11}$ A. Monsky, ${ }^{24}$ M. Nofrarias, ${ }^{14,15}$ S. Paczkowski, ${ }^{2}$ M. Perreur-Lloyd $\odot,{ }^{22}$ A. Petiteau, ${ }^{3}$ P. Pivato, ${ }^{6}$ E. Plagnol, ${ }^{3}$ J. Ramos-Castro, ${ }^{25,15}$ J. Reiche, ${ }^{2}$ F. Rivas, ${ }^{6}$ D. I. Robertson $\odot,{ }^{22, \S}$ G. Russano, ${ }^{6}$ J. Sanjuan, ${ }^{26}$ J. Slutsky, ${ }^{27}$ C. F. Sopuerta,,${ }^{14,15}$ F. Steier,${ }^{24}$ T. Sumner, ${ }^{19}$ D. Texier, ${ }^{10}$ J. I. Thorpe, ${ }^{27}$ D. Vetrugno, ${ }^{6}$ S. Vitale, ${ }^{6}$ V. Wand,${ }^{24}$ G. Wanner $\odot,{ }^{2, \ddagger}$ H. Ward $\odot,{ }^{22}$ P. J. Wass, ${ }^{19,20}$ W. J. Weber, ${ }^{6}$ L. Wissel ${ }^{2, \dagger}$ A. Wittchen, ${ }^{2}$ and P. Zweifel ${ }^{11}$

${ }^{1}$ European Space Technology Centre, European Space Agency, Keplerlaan 1, 2200 AG Noordwijk, The Netherlands

${ }^{2}$ Albert-Einstein-Institut, Max-Planck-Institut für Gravitationsphysik und Leibniz Universität Hannover, Callinstraße 38, 30167 Hannover, Germany

${ }^{3}$ APC, Université de Paris, CNRS, Astroparticule et Cosmologie, F-75006 Paris, France

${ }^{4}$ Department of Industrial Engineering, University of Trento, via Sommarive 9, 38123 Trento, Italy and Trento Institute for Fundamental Physics and Application/INFN, 38123 Povo, Trento, Italy

${ }^{5}$ Airbus Defence and Space, Claude-Dornier-Strasse, 88090 Immenstaad, Germany

${ }^{6}$ Dipartimento di Fisica, Università di Trento and Trento Institute for Fundamental Physics and Application/INFN, 38123 Povo, Trento, Italy

${ }^{7}$ Istituto di Fotonica e Nanotecnologie, CNR-Fondazione Bruno Kessler, I-38123 Povo, Trento, Italy

${ }^{8}$ DISPEA, Università di Urbino "Carlo Bo," Via Santa Chiara, 27, 61029 Urbino/INFN, Italy

${ }^{9}$ The School of Physics and Astronomy, University of Birmingham, B15 2TT Birmingham, United Kingdom

${ }^{10}$ European Space Astronomy Centre, European Space Agency, Villanueva de la Cañada, 28692 Madrid, Spain

${ }^{11}$ Institut für Geophysik, ETH Zürich, Sonneggstrasse 5, CH-8092 Zürich, Switzerland

${ }^{12}$ The UK Astronomy Technology Centre, Royal Observatory, Edinburgh, Blackford Hill, Edinburgh EH9 3 HJ, United Kingdom

${ }^{13}$ City University of Applied Sciences, Flughafenallee 10, 28199 Bremen, Germany

${ }^{14}$ Institut de Ciències de l'Espai (ICE, CSIC), Campus UAB, Carrer de Can Magrans s/n, 08193 Cerdanyola del Vallès, Spain

${ }^{15}$ Institut d'Estudis Espacials de Catalunya (IEEC), C/ Gran Capità 2-4, 08034 Barcelona, Spain

${ }^{16}$ isardSAT SL, Marie Curie 8-14, 08042 Barcelona, Catalonia, Spain

${ }^{17}$ Texas A\&M University, 701 H.R. Bright Building, College Station, Texas 77843-3141, USA

${ }^{18}$ European Space Operations Centre, European Space Agency, 64293 Darmstadt, Germany

${ }^{19}$ Physics Department, Blackett Laboratory, High Energy Physics Group, Imperial College London, Prince Consort Road, London SW7 2BW, United Kingdom

${ }^{20}$ Department of Mechanical and Aerospace Engineering, MAE-A, P.O. Box 116250, University of Florida, Gainesville, Florida 32611, USA

${ }^{21}$ Physik Institut, Universität Zürich, Winterthurerstrasse 190, CH-8057 Zürich, Switzerland

${ }^{22}$ SUPA, Institute for Gravitational Research, School of Physics and Astronomy, University of Glasgow, Glasgow G12 8QQ, United Kingdom

${ }^{23}$ Observatoire de la Côte d'Azur, Boulevard de l'Observatoire CS 34229-F 06304 Nice, France

${ }^{24} \mathrm{OHB}$ System AG, Universitätsallee 27-29, 28359 Bremen, Germany

${ }^{25}$ Department d'Enginyeria Electrònica, Universitat Politècnica de Catalunya, 08034 Barcelona, Spain

${ }^{26}$ Department of Physics, 2001 Museum Road, University of Florida, Gainesville, Florida 32611, USA

${ }^{27}$ Gravitational Astrophysics Laboratory, NASA Goddard Space Flight Center, 8800 Greenbelt Road, Greenbelt, Maryland 20771, USA

(Received 10 September 2020; accepted 12 February 2021; published 2 April 2021)

Published by the American Physical Society under the terms of the Creative Commons Attribution 4.0 International license. Further distribution of this work must maintain attribution to the author(s) and the published article's title, journal citation, and DOI. Open access publication funded by the Max Planck Society. 
We report on the first subpicometer interferometer flown in space. It was part of ESA's Laser Interferometer Space Antenna (LISA) Pathfinder mission and performed the fundamental measurement of the positional and angular motion of two free-falling test masses. The interferometer worked immediately, stably, and reliably from switch on until the end of the mission with exceptionally low residual noise of $32.0_{-1.7}^{+2.4} \mathrm{fm} / \sqrt{\mathrm{Hz}}$, significantly better than required. We present an upper limit for the sensor performance at millihertz frequencies and a model for the measured sensitivity above $200 \mathrm{mHz}$.

DOI: $10.1103 /$ PhysRevLett.126.131103

Introduction.-ESA's LISA Pathfinder (LPF) mission (launched December 3, 2015, ended June 30, 2017) was designed to prove that the technology needed for a spacebased gravitational wave detector like LISA [1] is feasible, in particular that test masses (TMs) could be placed in free fall with sufficiently low residual acceleration noise $[2,3]$. LPF's target was to achieve a differential acceleration noise between its two TMs within a factor of 10 of the LISA requirement. Critical to LPF's performance was the optical metrology system (OMS), which measured the TM-to-TM displacement interferometrically with a requirement of

$$
S_{\mathrm{OMS}}^{1 / 2}(f) \leq 9 \frac{\mathrm{pm}}{\sqrt{\mathrm{Hz}}} \sqrt{1+\left(\frac{3 \mathrm{mHz}}{f}\right)^{4}}
$$

over a frequency range of $1-30 \mathrm{mHz}$. This is a relaxation of the accuracy that will be required for the LISA TM interferometer by approximately 1 order of magnitude.

The OMS is directly comparable to the local interferometry required for LISA where a similar low-noise, multidegree-of-freedom readout is key to reaching the desired sensitivity. It can also be considered as a significant step in the development of optical interferometers in space, which could improve the accelerometer sensitivity in future geodesy missions, for example, $[4,5]$.

We report in this Letter that the OMS operated stably and reliably over the whole mission at a noise level significantly better than its design goal, showing that such a system can be constructed, aligned, integrated, and operated in space, a key milestone toward the construction of the more complicated LISA optical system. The performance of the LPF OMS allowed detailed modeling of the noise behavior of the first interferometric TM-to-TM displacement measurement system in free fall.

LISA Pathfinder: Optical metrology in space.-The LPF mission consisted of a single spacecraft on a Lissajous orbit around the Earth-Sun Lagrangian point $L 1$. It contained two free-falling TMs, each inside an electrode housing located in a small vacuum chamber. The position and orientation of both TMs along several degrees of freedom $(x, \phi, \eta$, see Fig. 2) was continuously monitored by the OMS, the high precision interferometric readout system. In science operations, the spacecraft operated in a "drag-free" state where one TM was allowed to freely follow its inertial path and the spacecraft and second TM were forced to follow the inertial TM. The spacecraft used the signals from the OMS to sense its own movement relative to the inertial mass and used these signals as one set of inputs to the dragfree and attitude control system. This system controlled 15 degrees of freedom of the relative position and attitude of the spacecraft and TMs through firing micro-Newton thrusters attached to the spacecraft and acting on the TMs through electrostatic forces.

The optical metrology system: The OMS consisted of the optical bench interferometer (OBI), reference laser unit, laser modulation unit, laser control unit, phasemeter, and data management unit [6-8]. A schematic is shown in Fig. 1. The OBI consisted of a $20 \times 21.2 \times 4.5 \mathrm{~cm}$ optical bench made of Zerodur onto which the optical components were hydroxide-catalysis bonded [9]. The OBI was located between the two vacuum chambers housing the TMs, and it was rigidly connected to the spacecraft. The laser light in the OBI originated in the reference laser unit, a Nd:YAG nonplanar ring oscillator that provided about $35 \mathrm{~mW}$ of single-mode light at $\lambda=1064 \mathrm{~nm}$. This light was fiber coupled into the laser modulator unit, where the beam was split by a $50 / 50$ beam splitter and each beam was then frequency shifted by an acousto-optic modulator (AOM) by about $80 \mathrm{MHz}$, such that a relative frequency shift of $f_{1}-f_{2}=f_{\text {het }}=1 \mathrm{kHz}$ was generated to enable heterodyne interferometry.

From the laser modulator unit, the light was then delivered via optical fibers to the OBI. On the optical bench, the beam paths were split into four different

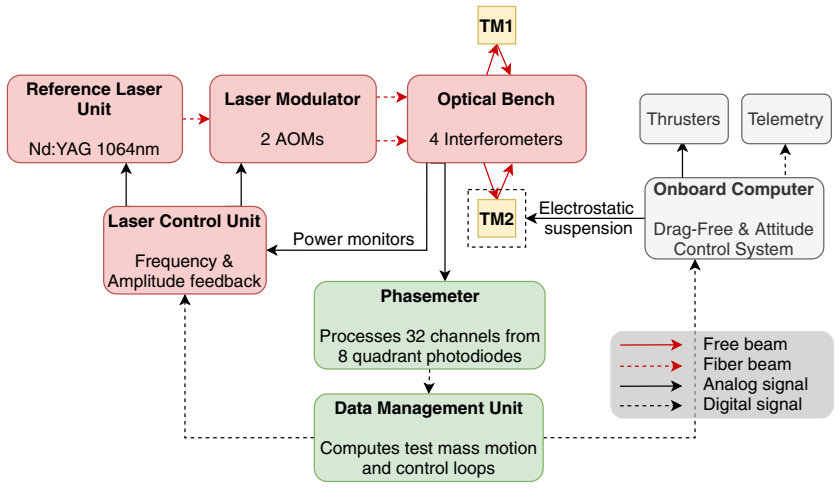

FIG. 1. A schematic overview of the OMS showing the linkages between the subsystems. The electrode housing around TM1 is not shown. 


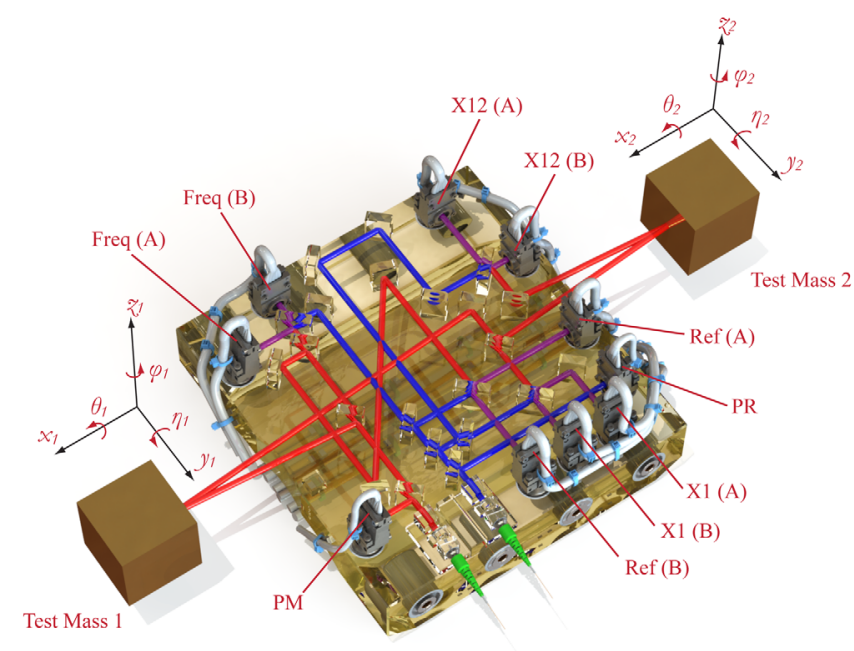

FIG. 2. The layout of the OBI showing the four interferometers (X12, X1, Ref., and Freq.) and their associated $A$ and $B$ photodiodes. They provide redundancy, allow subtraction of common noise, and improve the signal-to-noise ratio. The reference beam is blue, the measurement beam is red, and combined beams are purple. Two power monitor photodiodes (PM, PR) were used for sensing and stabilizing the laser beam power. Also shown are the TMs and their associated coordinate frames.

interferometers and finally detected by eight quadrant photo diodes. About $2 \mathrm{~mW}$ was reflected off TM1 and $1 \mathrm{~mW}$ off TM2. The signals were processed by two hotredundant phasemeter units, which performed single-bin discrete Fourier transforms [7] and passed data at $100 \mathrm{~Hz}$ to the data management unit. This computed linear and angular signal combinations, downsampled to $10 \mathrm{~Hz}$, and sent these signals to the onboard computer for use in the spacecraft and TM control and as science output data for downlink [10].

The optical bench interferometer: The OBI comprised four interferometers, which were needed in order to achieve the high precision readout: the so-called reference, frequency, $X 1$, and $X 12$ interferometers, see Fig. 2.

In the reference interferometer, both beams were routed entirely within the optical bench. The beams' optical path lengths were equal by design so that the reference interferometer sensed common mode noise sources that all interferometers were subject to, such as differential phase changes originating from the optical fibers or modulators. The reference interferometer signal $\left(x_{R}\right)$ was therefore subtracted from the all other interferometer readout signals to obtain clean measurements.

In the frequency interferometer, both beams were again routed entirely within the optical bench, however, the optical path lengths were intentionally unequal by $\sim 38 \mathrm{~cm}$, which enhanced the coupling of laser frequency noise. The phase readout signal of this interferometer had the reference signal subtracted and was then used in a digital control loop to stabilize the laser frequency (see [11]).
In the $X 1$ interferometer, the measurement beam (indicated in red in Fig. 2) reflected from TM1 before recombining with its reference beam (blue beam in Fig. 2) to give $x_{1}$. We then formed $o_{1}=x_{1}-x_{R}$, the displacement between the free-falling TM1 and the optical bench and, similarly, the angular signals $\eta_{1}$ and $\phi_{1}$. This interferometer readout was dominated by spacecraft motion, since the optical bench was rigidly connected to the spacecraft.

In the $X 12$ interferometer, the measurement beam reflected from both TMs before being recombined with its reference beam. We formed $o_{12}=x_{12}-x_{R}$ to get a signal that sensed differential displacement between both TMs and was therefore the main scientific readout signal in LPF. Similarly, we form the differential in- and out-ofplane angular readouts $\phi_{12}$ and $\eta_{12}$. By combining readouts, 1 longitudinal and 2 angular degrees of freedom were measured optically for each TM. The power of each beam was sensed by a dedicated power monitor photodiode. Their signals were used in an analog control loop for fast laser amplitude stabilization.

Results.-We present the amplitude spectral density (ASD) of the differential TM-to-TM displacement measurement in Fig. 3 and give an overview of its long-term mission behavior in Fig. 4 .

Figure 3 shows representative behavior under nominal conditions over the frequency range of $1 \mathrm{mHz}-5 \mathrm{~Hz}$. It highlights the modeled noise sources compared to the readout requirement. The total noise ASD was computed assuming all noises are uncorrelated.

We find that, at different frequencies, different noise sources dominate. Below $20 \mathrm{mHz}$ we are seeing genuine differential displacement noise between the TMs, largely driven by Brownian force noise on the TMs, as was shown in $[2,3]$.

With regards to intrinsic low-frequency noise of the OMS, there are three main thermally driven optical path length changes on the optical bench: the windows between the bench and the TMs [14], the expansion of the Zerodur baseplate, and the transmission through the beam splitters. We estimate an upper limit of thermally driven noise by taking the modeled thermal coupling, using an observed thermal measurement at $1.17 \mathrm{mHz}$ and a conservative projection to higher frequencies [15], and assuming no common mode rejection. Other thermoelastic induced noise was estimated to be below this level [16].

Between 20 and $200 \mathrm{mHz}$, the measured noise is increased due to tilt-to-length (TTL) coupling, i.e., the crosscoupling of lateral and angular jitter of the spacecraft and the TMs to the differential displacement readout signal $o_{12}[2,17]$.

Only above $200 \mathrm{mHz}$ OMS noise dominates the observed displacement noise. The most significant OMS noise sources are phasemeter readout noise, laser frequency noise, shot noise, and relative intensity noise (RIN). For nominal operating conditions, the first two were the main contributors, while shot noise and RIN were not limiting. 


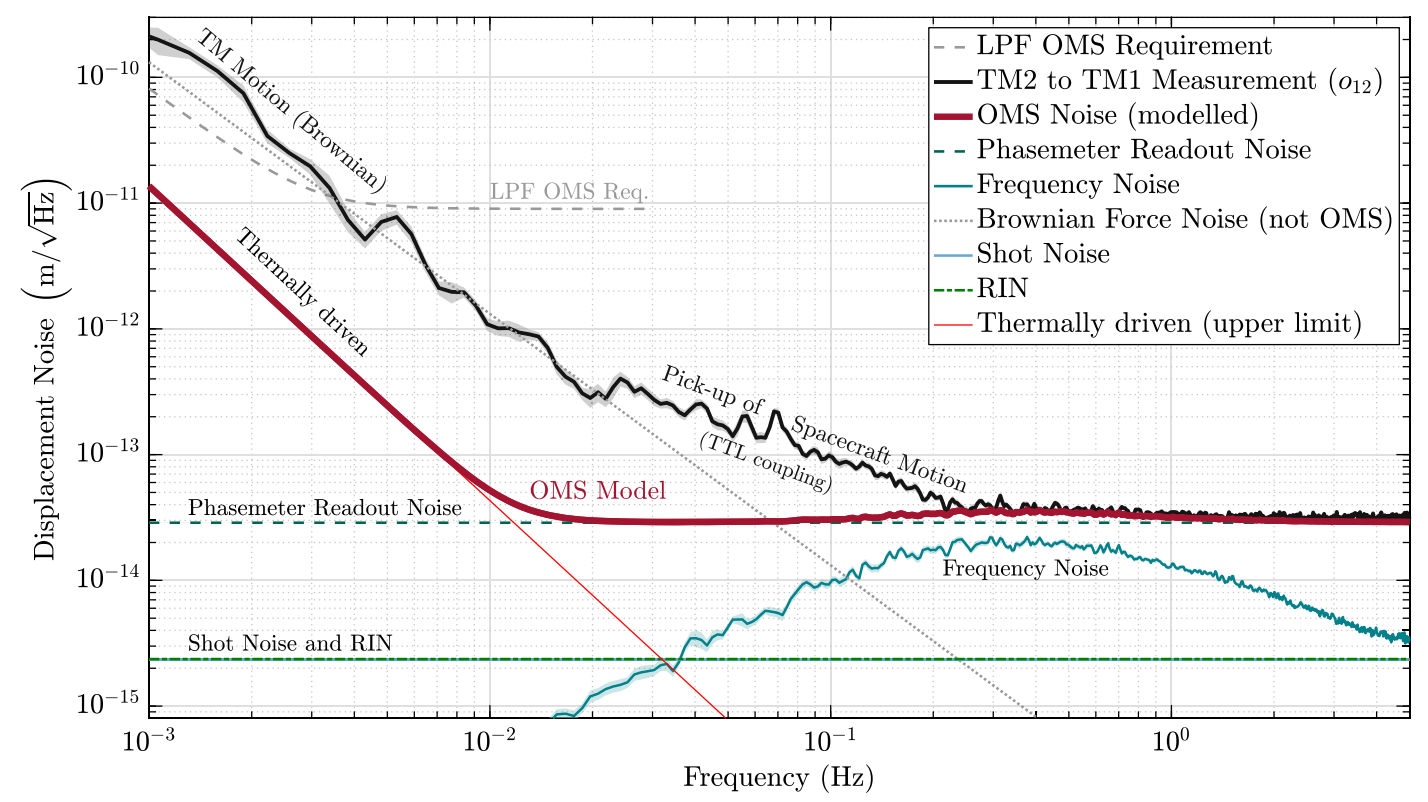

FIG. 3. ASD of the differential TM displacement noise $o_{12}$ on June 1, 2016, showing the performance of the OMS compared to its noise requirement and to the individual modeled OMS noise contributions. Above $200 \mathrm{mHz}$, the sum of the modeled OMS noise contributions is $29.7 \mathrm{fm} / \sqrt{\mathrm{Hz}}$, agreeing well with the observed level of $32.1 \mathrm{fm} / \sqrt{\mathrm{Hz}}$. Frequencies below $200 \mathrm{mHz}$ are dominated by other noise sources such as Brownian force noise and tilt-to-length coupling. We show an upper limit of the OMS sensing noise arising from temperature induced path length changes in transmissive optical components. This graph was generated using the LPSD algorithm [12] with 66.1\% overlapping BH92 windows [13], targeting 1000 Fourier frequencies with 100 averages.

The noise performance over the course of the mission is depicted in Fig. 4. Each blue data point corresponds to 12 min of data sampled with $10 \mathrm{~Hz}$, averaged over the nearly flat noise power spectral density frequency band between 1.2 and $2.8 \mathrm{~Hz}$. We have shown here only time segments where the spacecraft was in nominal operating mode without known interfering actions, such as the deliberate cooldown near the end of the mission. We see a bimodal noise distribution, which directly originates from two states of laser frequency noise. It can also be seen that June 1, 2016, used for Fig. 3, was representative (during a time with lower frequency noise).

This shows that, compared to the required $9 \mathrm{pm} / \sqrt{\mathrm{Hz}}$, the OMS operated stably and reliably with much lower noise levels at all given times between 27.8 and

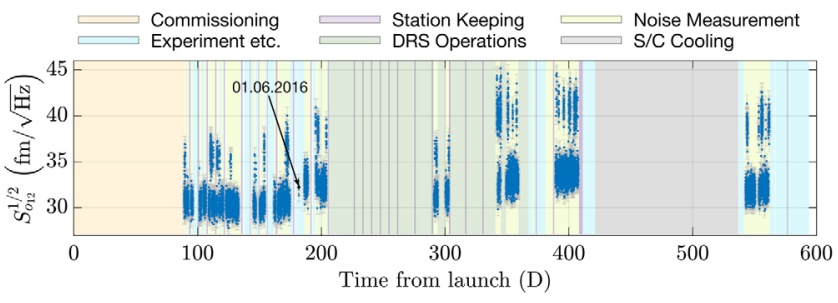

FIG. 4. OMS performance over mission duration. Shown are short-time ASD estimates of the differential displacement readout $o_{12}$ in the various mission phases, averaged between 1.2 and $2.8 \mathrm{~Hz}$. The data from Fig. 3 is marked by the date 01.06.2016. S/ C, spacecraft; DRS, disturbance reduction system.
$45.1 \mathrm{fm} / \sqrt{\mathrm{Hz}}$ and a median of $32.0_{-1.7}^{+2.4} \mathrm{fm} / \sqrt{\mathrm{Hz}}$. This marks the first ever long-term interferometric measurement with unprecedented sensitivity and frequency range in space.

Discussion.-We discuss the performance of the system and model the contributing noise sources during a representative period of 103 min starting at 18:46 UTC on June 1, 2016, see Fig. 3. During this time, the spacecraft was operating in a minimum noise configuration, with optimal configuration and no deliberate disturbances applied.

We performed a large number of OMS experiments to characterize different possible noise sources. A broader overview under varying operating conditions will be discussed in detail in a separate paper [10].

A comparison of the performance with the requirement is not directly possible, since OMS noise is not limiting in the $1-30 \mathrm{mHz}$ frequency band. At $20 \mathrm{mHz}$ and below, the overall noise is dominated by Brownian force noise. A very conservative comparison of the total noise at this frequency with the requirement shows that the OMS performed at very least a factor of 20 and up to a factor of 280 better than required.

Laser frequency noise: Laser frequency fluctuations couple directly into the phase measurement as described by $[18,19]$

$$
\delta \varphi=2 \pi \frac{\Delta L}{c} \delta \nu
$$


In this equation, $\delta \varphi$ are phase fluctuations measured in rad and $\Delta L$ is the path length mismatch between the reference and the measurement beam, $c$ denotes the speed of light, and $\delta \nu$ represents the laser frequency fluctuations measured in hertz.

The frequency interferometer was particularly sensitive to frequency noise due to its intentionally large path length mismatch $\Delta L_{F}-\Delta L_{R}=382 \mathrm{~mm}$ and was therefore used to estimate its contribution to $S_{O_{12}}$. By using Eq. (2) expressed as an ASD and accounting for the fact that the telemetry data from the $X 12$ and frequency interferometer had the reference interferometer signal subtracted, we find the corresponding displacement noise

$$
S_{o_{12}, \text { freq }}^{1 / 2}=\frac{\lambda}{4 \pi} \frac{\Delta L_{12}-\Delta L_{R}}{\Delta L_{F}-\Delta L_{R}} S_{\Psi_{F}}^{1 / 2}=: H_{o_{12}} \cdot S_{\Psi_{F}}^{1 / 2} .
$$

For the data in Fig. 3, the maximum frequency noise $S_{\Psi_{F}}^{1 / 2}$ was $(270 \pm 13) \mu \mathrm{rad} / \sqrt{\mathrm{Hz}}$ at $0.38 \mathrm{~Hz}$. The coupling coefficient $H_{o_{12}}$ was estimated by a dedicated experiment on the same day, giving $H_{o_{12}}=(82 \pm 1) \mathrm{pm} / \mathrm{rad}$, corresponding to a path length difference of $\Delta L_{12}-\Delta L_{R} \approx$ $368 \mu \mathrm{m}$, such that the resulting phase noise $S_{o_{12} \text {, freq }}^{1 / 2}$ had a maximum of $(22 \pm 1) \mathrm{fm} / \sqrt{\mathrm{Hz}}$.

We also experienced periods of slightly increased laser frequency noise, during which it dominated the $o_{12}$ measurement at frequencies between approximately 0.2 and $1 \mathrm{~Hz}$, causing the higher noise levels (bimodal distribution) visible in Fig. 4.

Shot noise: In the given context, we understand shot noise as statistical fluctuations of the incident photons on a photo diode and the resulting photo current, which then cause a phase noise. This noise occurs on every photodiode segment and therefore enters both in the longitudinal and angular readout signals. The phase noise in the longitudinal readout of interferometer $K \in\{X 12, X 1, R, F\}$ converted to an equivalent differential TM displacement noise is estimated by

$$
S_{K, \text { shot }}^{1 / 2}=\frac{\lambda}{2 \pi C_{K}} \sqrt{\frac{e}{2 \Sigma_{K} \frac{U_{\mathrm{ADC}}}{R_{\mathrm{TIA}}}},}
$$

where $C_{K}$ denotes the measured contrast and $e$ is the elementary charge. In-flight measurements provide us with a corresponding product term $\Sigma_{K}=\frac{1}{2} \varepsilon_{K}\left(P_{K, m}+P_{K, r}\right) \frac{R_{\mathrm{TIA}}}{U_{\mathrm{ADC}}}$, which represents the normalized photo current in the interferometer, with $\varepsilon_{K}$ being the effective photodiode responsivity and $P_{K ; m, r}$ denoting the power of the measurement and reference beam. We computed the shot noise trace in Fig. 3 by an uncorrelated sum of the contributing noises from the $X 12$ and $R$ interferometers using $C_{K}$ and $\Sigma_{K}$ telemetry, as well as the full range of the analog to digital convertor (ADC) $U_{\mathrm{ADC}}=5 \mathrm{~V}$ and the effective transimpedance $R_{\mathrm{TIA}}=6640 \Omega$. The resulting noise level of
$2.3 \mathrm{fm} / \sqrt{\mathrm{Hz}}$ can likewise be found if typical values are used instead of telemetry data: $\Sigma_{K} \approx 1.2, C_{K}>95 . \%$, $P_{K} \approx 1.2 \mathrm{~mW}$, and $0.7<\varepsilon_{K}<1 \mathrm{~A} / \mathrm{W}$.

Phasemeter noise: The phasemeter was the system that processed the photocurrent from the photodiode segments and produced an estimate of the phase of the $1 \mathrm{kHz}$ heterodyne signal. Each channel of the system consisted of a transimpedance amplifier, a low-pass filter, an ADC operating at $50 \mathrm{kHz}$ to digitize the signal, and a digital processing system that performed the demodulation to measure the phase.

The dominant noise sources are the equivalent input current noise and the quantization noise of the ADCs. Using LISO [20], the circuit was modeled to give an equivalent input current noise of $S_{I_{\text {elec }}}^{1 / 2}=17 \mathrm{pA} / \sqrt{\mathrm{Hz}}$. The remaining input current noise is attributed to the ADC and was estimated from ground measurements as $S_{I_{\mathrm{ADC}}}^{1 / 2}=$ $102 \mathrm{pA} / \sqrt{\mathrm{Hz}}$. From this the total input current noise $S_{I_{\mathrm{tot}}}$ is calculated and the equivalent displacement noise derived using

$$
S_{K, \text { phasemeter }}^{1 / 2}=\frac{\lambda}{\pi C_{K}} \frac{S_{I_{\mathrm{Iot}}}^{1 / 2}}{2 \Sigma_{K} \frac{U_{\mathrm{ADC}}}{R_{\mathrm{TIA}}}} .
$$

The total phasemeter noise contribution in the $o_{12}$ readout is computed by an uncorrelated sum of the contributing noises from the $X 12$ and $R$ interferometer. This gives an expected phasemeter noise level of $28.7 \mathrm{fm} / \sqrt{\mathrm{Hz}}$, which is the most significant contribution to the OMS noise budget at frequencies $>200 \mathrm{mHz}$ in the lower frequency noise state.

Laser RIN: RIN describes intensity fluctuations of a laser beam normalized by its mean. RIN results in instrument noise via three different mechanisms: a time varying direct force on the TMs (i.e., radiation pressure noise), coupling to phase noise at the heterodyne frequency $\left(1 f_{\text {het }}\right.$ coupling), and coupling to phase noise at twice the heterodyne frequency ( $2 f_{\text {het }}$ coupling).

The radiation pressure noise is suppressed well below the total noise by a power control loop and the $1 f_{\text {het }}$ coupling by the use of balanced detection (i.e., by subtracting the data retrieved from the $A$ and $B$ diodes).

The $2 f_{\text {het }}$ coupling is common mode in the $X 12$ and $R$ interferometers. This time there is no cancellation due to balanced detection. However, in normal operations, the control of the separation of the two TMs ensures that the path length difference $d_{o_{12}}$ between those interferometers is kept close to zero (e.g., $d_{o_{12}} \approx 0.3 \mathrm{~nm}$ in Fig. 3), which suppresses the effect described by

$$
S_{O_{12}, \mathrm{RIN}}^{1 / 2}=\frac{\lambda}{4 \pi} \sqrt{2} r \sin \left(\frac{4 \pi}{\lambda} d_{o_{12}}\right),
$$

where $r$ is the RIN level of the laser at $2 f_{\text {het }}$. 
Investigations of RIN coupling have been performed over a variety of operating conditions throughout the mission. From these, the estimated RIN in the frequency band above $0.8 \mathrm{~Hz}$ is approximately white and varies with time within the range from $r=1.8 \times 10^{-6}$ to $5.0 \times 10^{-6} / \sqrt{\mathrm{Hz}}$, probably due to variations in laser operating conditions. In total, well controlled RIN noise proved to be an insignificant OMS noise, contributing no more than $2.4 \mathrm{fm} / \sqrt{\mathrm{Hz}}$ during normal operation.

Conclusion.-The LPF OMS was the first heterodyne interferometer in space that measured the changes in separation and angle between two freely falling TMs housed within a drag-free spacecraft.

In flight the OMS performed stably and consistently much better than its requirement. At frequencies above $200 \mathrm{mHz}$ where the LPF performance was dominated by the OMS, a median noise level of $32.0_{-1.7}^{+2.4} \mathrm{fm} / \sqrt{\mathrm{Hz}}$ was observed.

The unprecedented performance allowed dedicated experiments to investigate the noise behavior in great detail, allowing the development and validation of a system noise model that approximates well the measured $o_{12}$ displacement noise above $200 \mathrm{mHz}$.

It demonstrates that a heterodyne interferometer implemented on a rigid, catalysis bonded platform using the demonstrated noise suppression techniques can sense multiple degrees of freedom at the level required for the LISA local interferometry.

This work has been made possible by the LISA Pathfinder mission, which is part of the space-science program of the European Space Agency. The AlbertEinstein-Institut acknowledges the support of the German Space Agency, DLR. The work is supported by the Federal Ministry for Economic Affairs and Energy based on a resolution of the German Bundestag (FKZ 50OQ0501 and FKZ 50OQ1601). The French contribution has been supported by the CNES (Accord Specific de projet CNES 1316634/CNRS 103747), the CNRS, the Observatoire de Paris, and the University Paris-Diderot. E. P. and H. I. would also like to acknowledge the financial support of the UnivEarthS Labex program at Sorbonne Paris Cité (ANR-10-LABX-0023 and ANR-11-IDEX0005-02). The Italian contribution has been supported by Agenzia Spaziale Italiana and Istituto Nazionale di Fisica Nucleare. The Spanish contribution has been supported by Contracts No. AYA2010-15709 (MICINN), No. ESP201347637-P, No. ESP2015-67234-P, and No. ESP2017-90084$\mathrm{P}$ (MINECO). Support from AGAUR (Generalitat de Catalunya) Contract No. 2017-SGR-1469 is also acknowledged. M. N. acknowledges support from Fundacion General CSIC (Programa ComFuturo). F. R. acknowledges an FPI contract from MINECO. The Swiss contribution acknowledges the support of the ETH Research Grant
No. ETH-05 16-2 and the Swiss Space Office (SSO) via the PRODEX Programme of European Space Agency. L. F. is supported by the Swiss National Science Foundation. The UK groups wish to acknowledge support from the United Kingdom Space Agency (UKSA), the Scottish Universities Physics Alliance (SUPA), the University of Glasgow, the University of Birmingham, and Imperial College London. J. I. T. and J.S. acknowledge the support of the U.S. National Aeronautics and Space Administration (NASA). N. K. is thankful for the support from a CNES Fellowship. The LISA Pathfinder Collaboration would like to acknowledge Prof. P.B., now deceased, Prof. J.A.L., now deceased, and L. G., now deceased, for their remarkable contribution to the LISA Pathfinder science.

\footnotetext{
*Deceased.

Corresponding author.

lennart.wissel@aei.mpg.de

Corresponding author.

gudrun.wanner@aei.mpg.de

${ }^{\S}$ Corresponding author.

david.robertson@glasgow.ac.uk
}

[1] P. Amaro-Seoane et al., Laser interferometer space antenna, https://arxiv.org/abs/1702.00786 (2017).

[2] M. Armano et al., Sub-Femto- $g$ Free Fall for Space-Based Gravitational Wave Observatories: LISA Pathfinder Results, Phys. Rev. Lett. 116, 231101 (2016).

[3] M. Armano et al., Beyond the Required LISA Free-Fall Performance: New LISA Pathfinder Results down to $20 \mu \mathrm{Hz}$, Phys. Rev. Lett. 120, 061101 (2018).

[4] G. Heinzel, F. G. Cervantes, A. F. G. Marín, J. Kullmann, W. Feng, and K. Danzmann, Deep phase modulation interferometry, Opt. Express 18, 19076 (2010).

[5] K.-S. Isleif, O. Gerberding, M. Mehmet, T. S. Schwarze, G. Heinzel, and K. Danzmann, Comparing interferometry techniques for multi-degree of freedom test mass readout, J. Phys. Conf. Ser. 716, 012008 (2016).

[6] G. Heinzel, C. Braxmaier, R. Schilling, A. Rüdiger, D. Robertson, M. te Plate, V. Wand, K. Arai, U. Johann, and K. Danzmann, Interferometry for the LISA technology package (LTP) aboard SMART-2, Classical Quantum Gravity 20, S153 (2003).

[7] G. Heinzel et al., The LTP interferometer and phasemeter, Classical Quantum Gravity 21, S581 (2004).

[8] C. Braxmaier et al., LISA Pathfinder optical interferometry, in Gravitational Wave and Particle Astrophysics Detectors, Proceedings of the Society of Photo-Optical Instrumentation Engineers (SPIE), edited by J. Hough and G. H. Sanders (SPIE, 2004), Vol. 5500, pp. 164-173, https://doi .org/10.1117/12.555266.

[9] A.-M. A. van Veggel and C. J. Killow, Hydroxide catalysis bonding for astronomical instruments, Adv. Opt. Technol. 3, 293 (2014).

[10] M. Armano et al., Sensor noise in LISA Pathfinder: An extensive in-flight review of the angular and longitudinal interferometric measurement system (to be published). 
[11] G. Hechenblaikner, V. Wand, M. Kersten, K. Danzmann, A. García, G. Heinzel, M. Nofrarias, and F. Steier, Digital laser frequency control and phase-stabilization loops in a high precision space-borne metrology system, IEEE J. Quantum Electron. 47, 651 (2011).

[12] M. Tröbs and G. Heinzel, Improved spectrum estimation from digitized time series on a logarithmic frequency axis, Measurement 39, 120 (2006).

[13] G. Heinzel, A. Rüdiger, and R. Schilling, Spectrum and spectral density estimation by the discrete Fourier transform (DFT), including a comprehensive list of window functions and some new flat-top windows (2002), http://hdl .handle.net/11858/00-001M-0000-0013-557A-5.

[14] M. Nofrarias, A. F. García Marín, A. Lobo, G. Heinzel, J. Ramos-Castro, J. Sanjuán, and K. Danzmann, Thermal diagnostic of the optical window on board LISA Pathfinder, Classical Quantum Gravity 24, 5103 (2007).

[15] M. Armano et al., Temperature stability in the sub-millihertz band with LISA Pathfinder, Mon. Not. R. Astron. Soc. 486, 3368 (2019).
[16] F. Gibert et al., Thermo-elastic induced phase noise in the LISA Pathfinder spacecraft, Classical Quantum Gravity 32, 045014 (2015).

[17] G. Wanner and N. Karnesis, Preliminary results on the suppression of sensing cross-talk in LISA Pathfinder, J. Phys. Conf. Ser. 840, 012043 (2017).

[18] H. Billing, K. Maischberger, A. Rudiger, R. Schilling, L. Schnupp, and W. Winkler, An argon laser interferometer for the detection of gravitational radiation, J. Phys. E 12, 1043 (1979).

[19] D. Robertson, C. Killow, H. Ward, J. Hough, G. Heinzel, A. Garcia, V. Wand, U. Johann, and C. Braxmaier, LTP interferometer-noise sources and performance, Classical Quantum Gravity 22, S155 (2005).

[20] G. Heinzel, Electronic noise in interferometers, in Gravitational Wave Detection II, Proceedings of the 2nd TAMA International Workshop, 1999 in Tokyo, Japan, edited by S. Kawamura and N. Mio (Universal Academy Press, Tokyo, 2000), Vol. 32, p. 83. 